\title{
The smoothness of orbital measures on noncompact symmetric spaces
}

\author{
Sanjiv Kumar Gupta and Kathryn E. Hare
}

\begin{abstract}
Let $G / K$ be an irreducible symmetric space where $G$ is a noncompact, connected Lie group and $K$ is a compact, connected subgroup. We use decay properties of the spherical functions to show that the convolution product of any $r=r(G / K)$ continuous orbital measures has its density function in $L^{2}(G)$ and hence is an absolutely continuous measure with respect to Haar measure. The number $r$ is approximately the rank of $G / K$. For the special case of the orbital measures, $\nu_{a_{i}}$, supported on the double cosets $K a_{i} K$ where $a_{i}$ belongs to the dense set of regular elements, we prove the sharp result that $\nu_{a_{1}} * \nu_{a_{2}} \in L^{2}$, except for the symmetric space of Cartan type $A I$ when the convolution of three orbital measures is needed (even though $\nu_{a_{1}} * \nu_{a_{2}}$ is absolutely continuous).
\end{abstract}

\section{Introduction}

Let $G$ be a real, connected, noncompact, semisimple Lie group with finite center, and $K$ a maximal compact subgroup of $G$. The quotient space, $G / K$, is a symmetric space of noncompact type, which we also assume to be irreducible. For $a \in G \backslash N_{G}(K)$, we let $\nu_{a}$ denote the $K$-bi-invariant, orbital, singular measure supported on the compact double coset $K a K$ in $G$. The smoothness properties of convolution products of these orbital measures has been of interest for many years and is related to questions about products of double cosets and spherical functions. Ragozin, in 21, proved that for $r \geq \operatorname{dim} G / K$, the convolution product measure, $\nu_{a_{1}} * \cdots * \nu_{a_{r}}$, is absolutely continuous with respect to any Haar measure on $G$, equivalently, its density function is a compactly supported function in $L^{1}(G)$. This was improved in a series of papers, culminating with [8] and [14, where $r$ was reduced to either $\operatorname{rank} G / K$ or $\operatorname{rank} G / K+1$ depending on the Lie type. See [11] for a good history of this problem.

For the special case of regular elements, $a_{j}$, it was shown in 2 that the density function of $\nu_{a_{1}} * \cdots * \nu_{a_{r}}$ belongs to the smaller space of compactly supported functions in $L^{2}(G)$ for $r \geq \operatorname{dim} G / K+1$. The decay properties of spherical functions and the Plancherel theorem were used to prove this. In this paper, we develop

2000 Mathematics Subject Classification. Primary 43A90; Secondary 43A85, 22E30.

Key words and phrases. symmetric space, orbital measure, spherical function.

This research is supported in part by NSERC 2016-03719 and by Sultan Qaboos University. The authors thank Acadia University for their hospitality when this research was done.

This paper is in final form and no version of it will be submitted for publication elsewhere. 
a more refined analysis of the decay properties of spherical functions, using the classification of these symmetric spaces in terms of their restricted root systems, to significantly improve this result. This analysis allows us to both extend the $L^{2}$ result to convolutions of all orbital measures $\nu_{a}$ for $a \notin N_{G}(K)$, as well as to reduce the number of convolution products to approximately $\operatorname{rank} G / K$; the precise values are given in Section 4 and depend only on the Lie and Cartan type of the symmetric space. In the special case of convolution products of orbital measures at regular elements, we prove that $r=2$ suffices, except for one symmetric space (Cartan type $A I$ of rank one) where $r=3$ is both necessary and sufficient. This latter fact shows that, unlike the situation for the analogous problem in compact Lie groups and algebras, it is not true that $\nu_{a}^{k}$ belongs to $L^{2}$ if and only if $\nu_{a}^{k}$ is absolutely continuous (where the exponent means convolution powers). The decay properties are also applied to study the differentiability of orbital measures.

\section{Notation and Basic Facts}

2.1. Lie algebra set up. Let $G$ be a real, connected, non-compact, semisimple Lie group with finite centre and let $K$ be a maximal compact subgroup of $G$ fixed by the Cartan involution $\theta$. We assume that $G / K$ is irreducible. The quotient space, $G / K$, is a symmetric space of non-compact type III in Helgason's terminology, [19. Let $\mathfrak{g}=\mathfrak{t} \oplus \mathfrak{p}$ be the corresponding Cartan decomposition of the Lie algebra $\mathfrak{g}$ of $G$, where $\mathfrak{t}$ is the Lie algebra of $K$ and $\mathfrak{p}$ is the orthogonal complement of $\mathfrak{t}$ with respect to the Killing form of $\mathfrak{g}$. We fix a maximal abelian (as a subalgebra of $\mathfrak{g}$ ) subspace $\mathfrak{a}$ of $\mathfrak{p}$ and let $\mathfrak{a}^{*}$ denote its dual. The rank of $G / K$ is the dimension of $\mathfrak{a}$. If we put $A=\exp \mathfrak{a}$ where exp: $\mathfrak{g} \rightarrow G$ is the exponential function, then $G=K A K$.

The set of restricted roots, $\Phi$, is defined by

$$
\Phi=\left\{\alpha \in \mathfrak{a}^{*}: \mathfrak{g}_{\alpha} \neq 0\right\}
$$

where $\mathfrak{g}_{\alpha}$ are the root spaces. The multiplicity of the restricted root $\alpha$ will be denoted

$$
m_{\alpha}=\operatorname{dim} \mathfrak{g}_{\alpha} .
$$

The subset of positive restricted roots is denoted $\Phi^{+}$. The set $\Phi$ is a root system, although not necessarily reduced as it is possible for both $\alpha$ and $2 \alpha$ to be in $\Phi$.

Take a basis $\mathcal{B}$ for $\mathfrak{a}^{*}$ consisting of positive simple roots and let $\mathfrak{a}^{+}$be the elements $H \in \mathfrak{a}$ with $\alpha(H)>0$ for all $\alpha \in \mathcal{B}$. Similarly, let $\mathcal{D} \subseteq \mathfrak{a}$ be the dual basis to $\mathcal{B}$ and let

$$
\mathfrak{a}^{*+}=\left\{\lambda \in \mathfrak{a}^{*}: \lambda(H)>0 \forall H \in \mathcal{D}\right\} .
$$

We have $\mathfrak{a}^{*}=\bigcup_{w \in W} w\left(\overline{\mathfrak{a}^{*+}}\right)$ for $W$ equal to the Weyl group, with a similar statement holding for $\mathfrak{a}$.

Consequently, $G=\overline{\operatorname{exp~a~}^{+}} K$. Indeed, given any $g \in G$, there is a pair $k_{1}, k_{2} \in K$ and a unique $X_{g} \in \overline{\mathfrak{a}^{+}}$such that $g=k_{1}\left(\exp X_{g}\right) k_{2}$. We can thus view $\lambda \in \mathfrak{a}^{*}$ as also acting on $g \in A$ by setting $\lambda(g)=\lambda\left(X_{g}\right)$.

The symmetric spaces can be classified by their Cartan class and the Lie type of their restricted root system, these being one of types $A_{n}, B_{n}, C_{n}, B C_{n}$, and $D_{n}$ (the classical types) or $G_{2}, F_{4}, E_{6}, E_{7}, E_{8}$ (the exceptional types), the subscript in all cases being the rank of the symmetric space. We remark that for types $B_{n}, C_{n}$ we may assume $n \geq 2$ as the symmetric spaces of Lie types $B_{1}$ and $C_{1}$ are isomorphic 
to type $A_{1}$. Similarly, with $D_{n}$ we may assume $n \geq 4$. For more details, please see the appendix.

For further background on this material and proofs of the facts stated above we refer the reader to [18]-[20].

2.2. Orbital measures. Next, we introduce the orbital measures of interest in this paper. We let $d m$ denote normalized Haar measure on $K$.

Definition 1. Let $a \in A$. By an orbital measure on $G$, we mean the measure denoted $\nu_{a}$, defined by the rule

$$
\int_{G} f(g) d \nu_{a}(g)=\int_{K} \int_{K} f\left(k_{1} a k_{2}\right) d m\left(k_{1}\right) d m\left(k_{2}\right)
$$

for all continuous, compactly supported functions $f$ on $G$.

The orbital measure $\nu_{a}$ is the $K$-bi-invariant, probability measure supported on the compact, double coset $K a K \subseteq G$. Orbital measures are always singular with respect to Haar measure on $G$ and they are continuous measures (i.e., have no atoms) when $a \notin N_{G}(K)$, the normalizer of $K$ in $G$.

It is a classical problem to study the smoothness of convolution products of continuous orbital measures. Some of the earliest work was done by Ragozin in 21 who showed that $\nu_{a_{1}} * \cdots * \nu_{a_{r}}$ is absolutely continuous if and only if the product of double cosets, $K a_{1} K a_{2} \cdots K a_{r} K$, has non-empty interior in $G$. He, then, used geometric arguments to prove that the latter statement was true whenever $r \geq \operatorname{dim} G / K$. Using algebraic methods, this was subsequently improved to $r \geq \operatorname{rank} G / K+1$ by Graczyk and Sawyer in $\mathbf{8}$, who also showed that this was sharp in the case of non-compact symmetric spaces with restricted root systems of type $A_{n}$. Inspired by Graczyk and Sawyer's work in [9] and 10, in [14 the authors proved that $r \geq \operatorname{rank} G / K$ is the sharp $L^{1}$ result for all the classical noncompact symmetric spaces except those of type $A_{n}$, and characterized precisely which convolution products are absolutely continuous for the classical types.

2.3. $L^{1}-L^{2}$ Dichotomy. Similar smoothness questions have been explored in a number of related settings, including $K$-bi-invariant measures supported on double cosets in compact symmetric spaces $G / K$, invariant measures supported on conjugacy classes of compact Lie groups or $A d$-invariant measures supported on adjoint orbits of compact Lie algebras. In the case of compact Lie groups and algebras, the authors in $\mathbf{1 2}$ and $\mathbf{1 3}$ used a combination of harmonic analysis and geometric arguments to show that convolution powers of such measures belong to $L^{1}$ if and only they belong to $L^{2}$, and determined the sharp exponent for each such measure. In contrast, in 3 it was shown that this dichotomy fails to hold in the compact symmetric space $S U(2) / S O(2)$.

The harmonic analysis approach to the $L^{2}$ problem for compact Lie groups involved studying the rate of decay of the characters of the group and applying the Plancherel theorem. For symmetric spaces, the analogous approach is to study, instead, the decay of the spherical transform. We recall the definition of the spherical function and spherical transform.

DEFINITION 2. The spherical transform of a compactly supported measure $\nu$ on the non-compact Lie group $G$ is defined by

$$
\widehat{\nu}(\lambda)=\int_{G} \phi_{\lambda}\left(g^{-1}\right) d \nu(g)
$$


where $\phi_{\lambda}$ is the spherical function corresponding to $\lambda \in \mathfrak{a}^{*}$ given by the expression

$$
\phi_{\lambda}(g)=\int_{K} \exp ((i \lambda-\rho) \mathcal{H}(g k)) d m(k) .
$$

Here $\rho$ is half the sum of the positive roots and $\mathcal{H}$ is the Iwasawa projection, i.e., $\mathcal{H}(g k)$ is the unique element in $\mathfrak{a}$ such that $g k \in K \exp \mathcal{H}(g k) N$ where $N$ is a Lie subgroup of $G$ with Lie algebra $\mathfrak{n}=\sum_{\alpha \in \Phi^{+}} \mathfrak{g}_{\alpha}$.

This formula for the spherical function can be found in [19, IV, Thm. 4.3] where it is also seen that $\phi_{\lambda}=\phi_{w(\lambda)}$ for all $w \in W$ and $\lambda \in \mathfrak{a}^{*}$.

From the definition of orbital measures it is easy to see that $\widehat{\nu_{a}}(\lambda)=\phi_{\lambda}\left(a^{-1}\right)$, while in $[2]$ it is shown that

$$
\left(\nu_{a_{1}} * \cdots * \nu_{a_{r}}\right)(\lambda)=\prod_{i=1}^{r} \phi_{\lambda}\left(a_{i}^{-1}\right) .
$$

A version of Plancherel's theorem holds in this setting. For the remainder of the paper, $c=c(\lambda)$ is the Harish-Chandra $c$ function and $d \lambda$ denotes Lebesgue measure on $\mathfrak{a}^{*}$.

TheOREM 1. (Plancherel) (see 19, IV Thm. 9.1]) The K-bi-invariant measure $\mu$ belongs to $L^{2}(G)$ if and only if

$$
\|\mu\|_{L^{2}(G)}^{2}=\int_{\mathfrak{a}^{*}}|\widehat{\mu}(\lambda)|^{2}|c(\lambda)|^{-2} d \lambda<\infty .
$$

Corollary 1 . The $k$-fold convolution product of the orbital measure $\nu_{a}$ belongs to $L^{2}(G)$ if and only if $\left|\phi_{\lambda}(a)\right|^{k}|c(\lambda)|^{-1} \in L^{2}\left(\mathfrak{a}^{*}\right)$.

It is known that the spherical functions have good decay properties. To explain, it is helpful to introduce further terminology and notation.

DEFINITIOn 3. (i) Given $a \in A$ (or $a \in \mathfrak{a}$ ), by the set of annihilating roots of a we mean the set

$$
\Phi(a)=\{\alpha \in \Phi: \alpha(a)=0\} .
$$

Put $\Phi^{+}(a)=\Phi(a) \cap \Phi^{+}$. By $\left(\Phi^{+}(a)\right)^{c}$ we mean the complement of $\Phi(a)$ in $\Phi^{+}$, that is, $\left(\Phi^{+}(a)\right)^{c}=\left\{\alpha \in \Phi^{+}: \alpha(a) \neq 0\right\}$.

(ii) If $\Phi(a)$ is empty, the element $a$ is called regular. If a is regular, we call $\nu_{a}$ a regular orbital measure.

We will let

$$
A_{0}=\left\{g \in A: g \notin N_{G}(K)\right\} .
$$

The set $N_{G}(K)$ can be characterized as the set of elements $g \in G$ such that $\alpha(g)=0$ for all roots $\alpha$, hence the set of annihilating roots of an element in $A_{0}$ is a proper root subsystem. The set of regular elements is dense in $A$ and in the special case of a rank one symmetric space all the elements of $A_{0}$ are regular.

Here is the decay result that we will use.

Proposition 1. (6. Thm. 11.1], see also [2 Prop. 4.1]) For each $a \in A_{0}$, there is a constant $C_{a}$ such that for all $\lambda \in \mathfrak{a}^{*}$,

$$
\left|\phi_{\lambda}(a)\right| \leq C_{a} \sum_{w \in W} \prod_{\alpha \in\left(\Phi^{+}(w(a))\right)^{c}}(1+|\langle\lambda, \alpha\rangle|)^{-m_{\alpha} / 2} .
$$


It is well known (see [19, IV.7.2 ]) that there is a constant $C$ such that

$$
|c(\lambda)|^{-1} \leq C \prod_{\alpha \in \Phi^{+}}(1+|\langle\lambda, \alpha\rangle|)^{m_{\alpha} / 2},
$$

thus

$$
\left(\left|\phi_{\lambda}(a)\right|^{k}|c(\lambda)|^{-1}\right)^{2} \leq\left. C_{a} \max _{w \in W} \prod_{\alpha \in\left(\Phi^{+}(w(a))\right)^{c}}|1+|\langle\lambda, \alpha\rangle||^{-m_{\alpha} k} \prod_{\alpha \in \Phi^{+}}|1+|\langle\lambda, \alpha\rangle\right|^{m_{\alpha}}
$$

for a new constant $C_{a}$. Combined with Plancherel's theorem, this implies $\nu_{a}^{k}$ belongs to $L^{2}(G)$ provided

$$
\int_{\overline{\mathfrak{a}^{*+}}} \max _{w \in W} \prod_{\alpha \in\left(\Phi^{+}(w(a))\right)^{c}}|1+\langle\lambda, \alpha\rangle|^{-m_{\alpha} k} \prod_{\alpha \in \Phi^{+}}|1+\langle\lambda, \alpha\rangle|^{m_{\alpha}} d \lambda<\infty .
$$

\section{3. $L^{2}$ results for convolutions of orbital measures at regular elements}

In [2], bounds were found for the right hand side of (2.2) that were sufficient to show that any convolution product of more than $\operatorname{dim} G / K$ regular orbital measures was in $L^{2}(G)$. We will begin by improving this result, in fact, obtaining sharp $L^{2}$ results for convolution products of regular orbital measures.

TheOREM 2. Suppose $a \in A_{0}$ is a regular element. The convolution products, $\nu_{a}^{k}$, belong to $L^{2}(G)$ if and only if $k \geq 2$, except if the symmetric space $G / K$ has restricted root system of type $A_{1}$ and is of Cartan class $A I$, in which case $k \geq 3$ is both necessary and sufficient.

REMARK 1. We remark that $k \geq 2$ is necessary since $\nu_{a}$ is always a singular measure.

We will first obtain bounds for $\left|\phi_{\lambda}(a)\right|^{k}|c(\lambda)|^{-1}$ for the symmetric spaces of classical Lie types. Let $\eta_{0}$ denote the multiplicity of the standard roots $e_{i} \pm e_{j}, \eta_{1}$ the multiplicity of the short roots $e_{i}$, and $\eta_{2}$ the multiplicity of the long roots $2 e_{i}$ (should there be roots of these forms). The reader can find the values of $\eta_{j}$ for each type in the appendix.

Lemma 1. Suppose the restricted root system of $G / K$ is one of the Lie types $A_{n}, B_{n}, C_{n}, B C_{n}$ or $D_{n}$ and that $a \in A_{0}$ is a regular element. There is a positive constant $C$, depending only on $G / K$ and $a$, such that

$$
\left(\left|\phi_{\lambda}(a)\right|^{k}|c(\lambda)|^{-1}\right)^{2} \leq C \min \left(1,\|\lambda\|^{(1-k) \varrho}\right) \text { for all } \lambda \in \mathfrak{a}^{*} \text { and } k \geq 1,
$$

where

$$
\varrho=\varrho(G / K)=\left\{\begin{array}{cc}
\eta_{0} n & \text { for Lie type } A_{n} \\
\eta_{0}(2 n-3)+\eta_{1}+\eta_{2} & \text { for Lie types } B_{n}, C_{n}, B C_{n}, n \geq 2 \\
\eta_{1}+\eta_{2} & \text { for Lie type } B C_{1} \\
\eta_{0} 2(n-1) & \text { for Lie type } D_{n}
\end{array} .\right.
$$

Proof. Throughout the proof, the constant $C$ may vary from one occurrence to another. We will assume $G / K$ has rank $n$ and there is no loss of generality in assuming $\lambda \in \overline{\mathfrak{a}^{*+}}$. 
As $a$ is regular, $\Phi(w(a))$ is empty for all $w \in W$ and thus

$$
\left(\left|\phi_{\lambda}(a)\right|^{k}|c(\lambda)|^{-1}\right)^{2} \leq C \prod_{\alpha \in \Phi^{+}}|1+\langle\lambda, \alpha\rangle|^{m_{\alpha}(1-k)} .
$$

Of course, if $\|\lambda\| \leq 1$, then $\prod_{\alpha \in \Phi^{+}}|1+\langle\lambda, \alpha\rangle| \leq C$, so our interest is in $\|\lambda\| \geq 1$.

We will let

$$
T_{\lambda}=\left\{\alpha \in \Phi^{+}:\langle\alpha, \lambda\rangle \geq c_{G}\|\lambda\|\right\}
$$

where the choice of constant $c_{G}>0$ will depend on the Lie type and will be made clear later in the proof. We will let $S_{0}=\left\{e_{i} \pm e_{j}: 1 \leq i<j \leq n\right\}$, $S_{1}=\left\{e_{i}: 1 \leq i \leq n\right\}$ and $S_{2}=\left\{2 e_{i}: 1 \leq i \leq n\right\}$ (should they exist). For example, in type $A_{n}, S_{0}=\Phi^{+}$and $S_{1}, S_{2}$ do not exist. Notice $m_{\alpha}=\eta_{j}$ if $\alpha \in S_{j}$. Put

$$
U_{\lambda, j}=T_{\lambda} \cap S_{j}
$$

and write $\left|U_{\lambda, j}\right|$ for the cardinality of this set.

With this notation, we have

$$
\left(\left|\phi_{\lambda}(a)\right|^{k}|c(\lambda)|^{-1}\right)^{2} \leq C \min \left(1,\|\lambda\|^{(1-k) \sum_{j} \eta_{j}\left|U_{\lambda, j}\right|}\right) .
$$

We will find lower bounds on $\left|U_{\lambda, j}\right|$ by analyzing on a type-by-type basis.

Type $A_{n}$ : We can write $\lambda=\sum_{i=1}^{n} a_{i} \lambda_{i}$ where $\lambda_{i}$ are the fundamental dominant weights (the dual basis to the basis of simple roots) and $a_{i} \geq 0$. Since all norms are equivalent on a finite dimensional normed space, we can take $\|\lambda\|=\max _{i} a_{i}=a_{m}$ (say). It will suffice to determine which positive roots $\alpha=\sum_{i=1}^{n} b_{i} \alpha_{i}$ have $b_{m}>0$ (and hence $b_{m} \geq 1$ ) for then $\langle\alpha, \lambda\rangle=\sum_{i} a_{i} b_{i} \geq a_{m} b_{m} \geq\|\lambda\|$ and $U_{\lambda, 0}$ will contain that set of roots. (Here we will take $c_{G}=1$.) These will be the roots $\alpha=e_{i}-e_{j}$ where $1 \leq i \leq m$ and $m<j \leq n+1$, thus the minimum value of $\left|U_{\lambda, 0}\right|$ is $n$.

Type $B_{n}, C_{n}, B C_{n}$ : We leave the very easy case of $B C_{1}$ to the reader and assume $n \geq 2$. Here we can write $\lambda=\sum_{i=1}^{n} a_{i} e_{i}$ where $a_{i} \geq 0$ are non-increasing, and $e_{i}$ are the standard basis vectors for $\mathbb{R}^{n}$. Taking the Euclidean norm, we have $a_{1} \leq\|\lambda\| \leq n a_{1}$. We have $\langle\alpha, \lambda\rangle \geq a_{1}$ if $\alpha=e_{1}+e_{j}$ for $j=2, \ldots, n$ or $\alpha=(2) e_{1}$. In particular, for any choice of $c_{G} \leq 1$ we have $\left|U_{\lambda, j}\right| \geq 1$ for $j=1$ in type $B_{n}$, for $j=2$ for type $C_{n}$ and for both $j=1,2$ for type $B C_{n}$. If $a_{2} \leq a_{1} / 2$, then we also have $\langle\alpha, \lambda\rangle \geq a_{1} / 2$ if $\alpha=e_{1}-e_{j}$ for $j=2, \ldots, n$. In this case, $\left|U_{\lambda, 0}\right| \geq 2(n-1)$. Otherwise, $a_{2}>a_{1} / 2$, and then $\langle\alpha, \lambda\rangle \geq a_{1} / 2$ if $a=e_{2}+e_{j}, j=3, \ldots, n$ or $\alpha=(2) e_{2}$. In this case, taking $c_{G}=1 / 2$, we have $\left|U_{\lambda, 0}\right| \geq 2 n-3$ and $\left|U_{\lambda, 1}\right| \geq 2$ for type $B_{n}$, with similar statements for $C_{n}$ and $B C_{n}$. Of course, the minimum of $2 \eta_{0}(n-1)+\eta_{1}+\eta_{2}$ and $\eta_{0}(2 n-3)+2\left(\eta_{1}+\eta_{2}\right)$ is at least $\eta_{0}(2 n-3)+\left(\eta_{1}+\eta_{2}\right)$.

Type $D_{n}$ : As with type $A_{n}$, we write $\lambda=\sum_{i=1}^{n} a_{i} \lambda_{i}$ where $\lambda_{i}$ are the fundamental dominant weights and $a_{i} \geq 0$. We again take $c_{G}=1$. It suffices to determine which $\alpha=\sum b_{i} \alpha_{i}$ have $b_{m}>0$, where $a_{m}=\max _{i} a_{i}$. If $m \neq n-1, n$, these will be the roots $\alpha=e_{i}+e_{j}$ for $i \leq m$ and $j>i$ and for $\alpha=e_{i}-e_{j}$ for $i \leq m<j$. There are at least $2(n-1)$ of these roots. If $m=n$, all the roots $e_{i}+e_{j}$ have the desired property, while if $m=n-1$, the positive roots $e_{i}-e_{n}, i<n$ and $e_{i}+e_{j}, i<j<n$ all work. Thus for all $\lambda,\left|U_{\lambda, 0}\right| \geq \min \left(2(n-1),\left(\begin{array}{l}n \\ 2\end{array}\right)\right)=2(n-1)$ as we may assume $n \geq 4$ for this type.

Proof. [of Theorem 2] We begin by proving the sufficiency of the choice of $k$. As in the lemma, the constant $C>0$, depending on $G / K$ and $a$, which appears 
throughout may change from one occurrence to another. We again assume $G / K$ has rank $n$.

When $G / K$ has a restricted root space of classical Lie type, the previous lemma shows that

$$
\left\|\nu_{a}^{k}\right\|_{2}^{2} \leq C \int_{\overline{\mathfrak{a}}^{*+}} \min \left(1,\|\lambda\|^{(1-k) \varrho}\right) d \lambda \leq C \int_{1}^{\infty} t^{(1-k) \varrho} t^{n-1} d t
$$

and this will be finite if $(1-k) \varrho+n-1<-1$. It is a routine exercise, using the values of $\varrho$ given in the Lemma, to see that if $k \geq 2$, then this is true for all these classical types, except if $G / K$ is of Lie type $A_{n}$ and Cartan class $A I$. In this latter case, $\eta_{0}=1$ and we have that the integral above is finite provided $k \geq 3$.

However, the argument can be improved for the Lie type $A_{n}$, Cartan class $A I$, when $n \geq 2$. Let

$$
\begin{aligned}
& \Lambda_{0}=\left\{\lambda=\sum_{i=1}^{n} a_{i} \lambda_{i} \in \overline{\mathfrak{a}^{*+}}: a_{j}=\max a_{i} \text { for some } j \neq 1, n\right\}, \\
& \Lambda_{1}=\left\{\lambda=\sum_{i=1}^{n} a_{i} \lambda_{i} \in \overline{\mathfrak{a}^{*+}}: a_{1}=a_{n}=\max a_{i}\right\}
\end{aligned}
$$

and let $\Lambda_{2}$ be the rest of $\overline{\mathfrak{a}^{*+}}$. Note that

$$
\left\|\nu_{a}^{2}\right\|_{2}^{2} \leq C \sum_{j=0}^{2} \int_{\Lambda_{j}}\left(\left|\phi_{\lambda}(a)\right|^{2}|c(\lambda)|^{-1}\right)^{2} d \lambda .
$$

Let $U_{\lambda, 0}$ be as in the lemma. Note that $\left|U_{\lambda, 0}\right| \geq n+1$ if $\lambda \in \Lambda_{0} \cup \Lambda_{1}$, from whence one can see that $\int_{\Lambda_{j}}\left(\left|\phi_{\lambda}(a)\right|^{2}|c(\lambda)|^{-1}\right)^{2} d \lambda<\infty$ for $j=0,1$.

If, instead $\lambda \in \Lambda_{2}$ (so either $a_{1}$ or $a_{n}$ is the unique maximal coordinate), then $\left|U_{\lambda, 0}\right|=n$. However, there will also be at least $n-1$ positive roots $\alpha \notin U_{\lambda, 0}$ such that $\langle\alpha, \lambda\rangle \geq a_{J}$, where $a_{J}$ is the second largest coefficient. Using this fact, we obtain the bound

$$
\begin{aligned}
\int_{\Lambda_{2}}\left(\left|\phi_{\lambda}(a)\right|^{2}|c(\lambda)|^{-1}\right)^{2} d \lambda & \leq C \int_{0}^{\infty}\left(1+t_{1}\right)^{-n}\left(\int_{0}^{t_{1}}\left(1+t_{2}\right)^{-(n-1)} t_{2}^{n-2} d t_{2}\right) d t_{1} \\
& \leq C\left(1+\int_{1}^{\infty} t_{1}^{-n} \int_{1}^{t_{1}} t_{2}^{-1} d t_{1}\right) \\
& =C\left(1+\int_{1}^{\infty} t_{1}^{-n} \log t_{1} d t_{1}\right)
\end{aligned}
$$

and this is finite since we are assuming $n \geq 2$.

Thus even when the symmetric space is of Cartan class $A I$, we have $\nu_{a}^{2} \in L^{2}$ provided the rank of $G / K$ is at least $n=2$. That completes the proof of sufficiency of the choice of $k$ for the classical Lie types.

For the symmetric spaces with restricted root spaces of exceptional Lie types, we argue in a similar fashion. We define $T_{\lambda}$ as in (3.2) and decompose the set of positive restricted roots into maximal disjoint sets $S_{j}$, consisting of the positive roots of a given multiplicity. Again, put $U_{\lambda, j}=T_{\lambda} \cap S_{j}$ and observe that again (3.4) holds.

If the restricted root space is Lie type $G_{2}, E_{6}, E_{7}$ or $E_{8}$, then all the roots have the same multiplicity, so we take $S_{0}=\Phi^{+}$. It is shown in $1 \mathbf{1 5}$ (see, for 
example, Tables 2,3,4) that the minimum cardinality of $U_{\lambda, 0}$ is at least 5,16,27 and 57 respectively.

If the restricted root space is Lie type $F_{4}$ and all the roots have the same multiplicity, again $S_{0}=\Phi^{+}$and the minimum cardinality of $U_{\lambda, 0}$ is shown in [15] to be 15. Otherwise, there are two distinct multiplicities and we define $S_{0}, S_{1}$ accordingly. As can be seen from [15, $\left|U_{\lambda, 0}\right| \geq 9$ and $\left|U_{\lambda, 1}\right| \geq 6$. Using (3.5) again, it is easy to check that $k \geq 2$ suffices.

We turn now to proving the necessity of the choice of $k$. Since $\nu_{a}$ is a singular measure with respect to Haar measure, $k \geq 2$ is certainly necessary (in all cases). Thus we need only consider the symmetric space $G / K$ of Lie type $A_{1}$ and Cartan class $A I$ and show that $\nu_{a}^{2}$ does not belong to $L^{2}$.

For this symmetric space, the spherical functions can be expressed in terms of the hypergeometric functions ${ }_{2} F_{1}$ as follows. Denote by $\alpha$ the (single) positive root and choose $H_{0} \in \mathfrak{a}$ such that $\alpha\left(H_{0}\right)=1$. For any $t \neq 0$, it is known $([\mathbf{2 2}, 11.5 .15])$ that

$$
\phi_{\lambda}\left(\exp t H_{0}\right)={ }_{2} F_{1}\left(\frac{1+i \lambda}{4}, \frac{1-i \lambda}{4}, 1,-\sinh ^{2} t\right) .
$$

Next, we use the relationship between the hypergeometric functions and the Jacobi and Bessel functions (c.f., [7, Sec. 6.4]):

$$
J_{u}^{(0, b)}(t)={ }_{2} F_{1}\left(\frac{b+1+i u}{2}, \frac{b+1-i u}{2}, 1,-\sinh ^{2} t\right),
$$

while

$$
J_{u}^{(0, b)}(t)=c J_{0}(u t)+O\left(u^{-3 / 2}\right),
$$

where $J_{0}(\cdot)$ is the Bessel function and $c$ is a non-zero constant depending on $t$. It is well known ([1,9.2.1]) that for $z>0$,

$$
J_{0}(z)=\frac{C}{\sqrt{z}}\left(\cos (z-\pi / 4)+O\left(z^{-1}\right)\right)
$$

for some $C \neq 0$. Thus for all $\lambda>0$,

$$
\phi_{\lambda}\left(\exp t H_{0}\right)=\frac{C}{\sqrt{\lambda}} \cos (\lambda t / 2-\pi / 4)+O\left(|\lambda|^{-3 / 2}\right)
$$

where the non-zero constant $C$ depends only on $t$.

For any integer $j$, let $I_{j}$ denote the interval

$$
I_{j}=\frac{2}{t}\left[(2 j+1) \frac{\pi}{2}+\frac{\pi}{8},(2 j+1) \frac{\pi}{2}+\frac{3 \pi}{8}\right]
$$

and let $\bigcup_{j \in \mathbb{Z}} I_{j}=I^{*}$. If $\lambda \notin I^{*}$, then $|C \cos (\lambda t / 2-\pi / 4)| \geq|C \cos 3 \pi / 8|=\varepsilon_{0}>0$. The asymptotic formula for $\phi_{\lambda}$, (3.6), shows that we may choose $\lambda_{1}$ sufficiently large so that for all $\lambda \geq \lambda_{1}$ with $\lambda \notin I^{*}$, we have

$$
\left|\phi_{\lambda}\left(\exp t H_{0}\right)\right| \geq \frac{\varepsilon_{0}}{2 \sqrt{\lambda}} .
$$

It is shown in the proof of Prop. 7.2 in [19], that for the Harish Chandra $c$ function, $\lim _{\lambda \rightarrow \infty} c(\lambda)^{-1} \lambda^{-1 / 2}=2 \sqrt{\pi}$. Thus $c(\lambda)^{-1} \geq \sqrt{\pi \lambda}$ for all $\lambda \geq \lambda_{2}$, say. Let $\lambda_{0}=$ $\max \left(\lambda_{1}, \lambda_{2}\right)$. Putting these bounds together shows that

$$
\int\left|\phi_{\lambda}^{2}\left(\exp t H_{0}\right) c(\lambda)^{-1}\right|^{2} d \lambda \geq \int_{\lambda \notin I^{*}, \lambda \geq \lambda_{0}}\left(\frac{\varepsilon_{0}}{2 \sqrt{\lambda}}\right)^{4}(\sqrt{\pi \lambda})^{2} d \lambda=C \int_{\lambda \notin I^{*}, \lambda \geq \lambda_{0}} \frac{d \lambda}{\lambda} .
$$


Choose $k_{0}$ such that $(2 k-1) \pi / 2+3 \pi / 8 \geq \lambda_{0}$ for $k \geq k_{0}$ and set

$$
L_{k}=\frac{2}{t}\left[(2 k-1) \frac{\pi}{2}+\frac{3 \pi}{8},(2 k+1) \frac{\pi}{2}+\frac{\pi}{8}\right] .
$$

We deduce that

$$
\int\left|\phi_{\lambda}^{2}\left(\exp t H_{0}\right) c(\lambda)^{-1}\right|^{2} d \lambda \geq C \sum_{k=k_{0}}^{\infty} \int_{L_{k}} \frac{d \lambda}{\lambda} \geq C \sum_{k=k_{0}}^{\infty} \frac{\text { length }\left(L_{k}\right)}{k}=\infty .
$$

Consequently, $\phi_{\lambda}^{2}\left(\exp t H_{0}\right) c(\lambda)^{-1} \notin L^{2}$ and that proves $\nu_{a}^{2} \notin L^{2}$ for any $a=$ $\exp t H_{0}, t \neq 0$, and hence for any regular $a$.

REMARK 2. It is known that for any non-compact, rank 1 symmetric space, $\nu_{a} * \nu_{a}$ belongs to $L^{1}$ for all $a \in A_{0}(\mathbf{8})$. Thus the $L^{1}-L^{2}$ dichotomy fails for the symmetric space of Lie type $A_{1}$ and Cartan class AI. Interestingly, the $L^{1}-$ $L^{2}$ dichotomy holds for all the regular orbital measures in all the other symmetric spaces since we obviously have $\nu_{a}^{k} \in L^{1}$ only if $k \geq 2$.

Corollary 2. Let $a_{1}, a_{2}, a_{3}$ be regular elements in $A$. If $G / K$ is Lie type $A_{1}$ and Cartan class AI, then $\nu_{a_{1}} * \nu_{a_{2}} * \nu_{a_{3}} \in L^{2}$. Otherwise, $\nu_{a_{1}} * \nu_{a_{2}} \in L^{2}$.

Proof. We will prove the first statement as the second is even easier. Let $\mu=$ $\nu_{a_{1}} * \nu_{a_{2}} * \nu_{a_{3}}$. By the Plancherel formula,

$$
\|\mu\|_{2}^{2}=\int_{\mathfrak{a}^{*}}|\widehat{\mu}(\lambda)|^{2}|c(\lambda)|^{-2} d \lambda=\int_{\mathfrak{a}^{*}}\left|\prod_{i=1}^{3} \phi_{\lambda}\left(a_{i}^{-1}\right)\right|^{2}|c(\lambda)|^{-2} d \lambda .
$$

Applying the generalized Holder's inequality gives

$$
\|\mu\|_{2}^{2} \leq \prod_{i=1}^{3}\left(\int_{\mathfrak{a}^{*}}\left|\phi_{\lambda}\left(a_{i}^{-1}\right)\right|^{6}|c(\lambda)|^{-2} d \lambda\right)^{1 / 3}=\prod_{i=1}^{3}\left\|\nu_{a_{i}}^{3}\right\|_{2}^{2 / 3}
$$

and the latter product is finite according to the Theorem.

\section{Smoothness of convolutions of arbitrary orbital measures}

4.1. $L^{2}$ results. The goal of this section is to show that for all $a \in A_{0}$ (not just regular $a$ ) there is an index $k$ such that $\nu_{a}^{k} \in L^{2}(G)$. As in the proof of Theorem 2. we will continue to use the notation $\eta_{0}$ to denote the multiplicity of the roots $e_{i} \pm e_{j}, \eta_{1}$ for the multiplicity of the short roots $e_{i}$, and $\eta_{2}$ for the multiplicity of the long roots $2 e_{i}$ when the symmetric space is of classical Lie type $A_{n}, B_{n}, C_{n}$, $B C_{n}$ or $D_{n}$. We recall that the values of $\eta_{j}$ depend on the Lie type and Cartan class and can be found in the Appendix.

TheOREM 3. Let $G / K$ be a non-compact symmetric space of type $A_{n}, B_{n}$, $C_{n}, D_{n}$ or $B C_{n}$. If $v_{a_{1}}, \ldots, v_{a_{k}}$ are any orbital measures on $G$ with $a_{i} \in A_{0}$, then $v_{a_{1}} * \cdots * v_{a_{k}} \in L^{2}(G)$ provided $k>k_{G}$ where

$$
k_{G}=\left\{\begin{array}{cc}
n+n / \eta_{0} & \text { for type } A_{n} \\
n-1+n /\left(2 \eta_{0}\right) & \text { for type } D_{n} \\
2(n-1)+\left(n+\eta_{1}+\eta_{2}\right) / \eta_{0} & \text { for type } B_{n}, C_{n}, B C_{n}, n \geq 3 \\
\max \left(4,2+\left(\eta_{1}+\eta_{2}\right) /\left(2 \eta_{0}\right)\right) & \text { for } B_{2}, C_{2}, B C_{2}
\end{array} .\right.
$$


REMARK 3. We remark that the symmetric spaces of Lie type $A_{n},(B) C_{n}$ or $D_{n}$ have rank $n$ and dimension $O\left(n\left(n+\eta_{1}+\eta_{2}\right)\right)$. Note that for type $(B) C_{n}$ we can assume $n \geq 2$ as the regular orbital measure case has already be done.

The key to the proof of this theorem is finding bounds for the products

$$
P_{G / K}^{w}(\lambda, k, a)=\prod_{\alpha \in \Phi^{+}(w(a))^{c}}|1+\langle\lambda, \alpha\rangle|^{-m_{\alpha} k} \prod_{\alpha \in \Phi^{+}}|1+\langle\lambda, \alpha\rangle|^{m_{\alpha}},
$$

and

$$
P_{G / K}(\lambda, k, a)=\max _{w \in W} P_{G / K}^{w}(\lambda, k, a)
$$

for $\lambda \in \overline{\mathfrak{a}^{*+}}$ since we have already seen in (2.2) that

$$
\left(\left|\phi_{\lambda}(a)\right|^{k}|c(\lambda)|^{-1}\right)^{2} \leq C_{a} P_{G / K}(\lambda, k, a) .
$$

This will be mainly accomplished in two lemmas. We will again write $C$ for a positive constant (depending only on $G / K$ and $a$ ) that may change throughout the proof. We begin with the symmetric spaces of Lie type $A_{n}$ or $D_{n}$. These are easier as all roots have the same multiplicity.

Lemma 2. Suppose $G / K$ is Lie type $A_{n-1}$ or $D_{n}$ and $a \in A_{0}$. There is a constant $C$ such that

$$
P_{G / K}(\lambda, k, a) \leq C \min \left(1,\|\lambda\|^{\left.-\eta_{0} p_{k}\right)}\right)
$$

for all integers $k \geq n-1$ and $\lambda \in \overline{\mathfrak{a}^{*+}}$, where

$$
p_{k}=p_{k}(G / K)=\left\{\begin{array}{cc}
k-n+1 & \text { for } G / K \text { type } A_{n-1} \\
2(k-n+1) & \text { for } G / K \text { type } D_{n}
\end{array} .\right.
$$

Proof. There is a constant $C$ such that $P_{G / K}^{w}(\lambda, k, a) \leq C$ if $\|\lambda\| \leq 1$, thus our interest is with $\|\lambda\| \geq 1$.

In [16] the analogous problem was studied for the invariant measures supported on conjugacy classes in the classical simple compact Lie groups. Specifically, in (3.1) of [16], it was shown that if $\mathcal{G}$ is a compact Lie group, $X^{+}$is the set of positive roots for the Lie algebra associated with $\mathcal{G}$ and $Y^{+}$is the set of positive roots of some maximal root subsystem, then for all representations $\lambda$,

$$
\prod_{\alpha \in\left(Y^{+}\right)^{c}}|1+\langle\lambda, \alpha\rangle|^{-1} \leq C \prod_{\alpha \in X^{+}}|1+\langle\lambda, \alpha\rangle|^{-s}
$$

where $s=1 /(n-1)$ if $\mathcal{G}$ is Lie type $A_{n-1}$ or $D_{n}$. Athough this was formally shown only for all representations of $\mathcal{G}$, the same reasoning gives the same bound for all $\lambda \in \overline{\mathfrak{a}^{*+}}$ with $\|\lambda\| \geq 1$.

Consider the compact Lie group $\mathcal{G}$ with the same root system $\Phi$ as the restricted root system of $G / K$ (although, with all roots having multiplicity two, rather than $\left.\eta_{0}\right)$. For any $a \in A_{0}$ and $w \in W$, the set of positive annihilating roots of $w(a)$ is contained in the set of positive roots of a maximal root subsystem of $\Phi$, say $\Psi^{+}$. Appealing to (4.2) we deduce that if $\|\lambda\| \geq 1$, then

$$
\begin{aligned}
P_{G / K}^{w}(\lambda, k, a) & \leq\left(\prod_{\alpha \in\left(\Psi^{+}\right)^{c}}|1+\langle\lambda, \alpha\rangle|^{-k} \prod_{\alpha \in \Phi^{+}}|1+\langle\lambda, \alpha\rangle|\right)^{\eta_{0}} \\
& \leq C \prod_{\alpha \in \Phi^{+}}|1+\langle\lambda, \alpha\rangle|^{(1-k s) \eta_{0}}
\end{aligned}
$$


(for the appropriate choice of $s$ ). Hence, if we let $q$ be the minimal number of positive roots $\alpha$ (not counting multiplicity) such that $\langle\lambda, \alpha\rangle \geq c_{G}\|\lambda\|$ for such $\lambda$, with $c_{G}>0$ as in (3.2), then $P_{G / K}(\lambda, k, a) \leq C\|\lambda\|^{(1-k s) \eta_{0} q}$. In the notation of (3.3), $q=\min _{\lambda}\left|U_{\lambda, 0}\right|$. Thus $q\left(A_{n-1}\right)=n-1$ and $q\left(D_{n}\right)=2(n-1)$. Inputting the values for $s$ and $q$ gives the desired result.

Lemma 3. Suppose $G / K$ is Lie type $B_{n}, C_{n}$ or $B C_{n}, \lambda \in \overline{\mathfrak{a}^{*+}}$ and $a \in A_{0}$.

(i) If $n \geq 3$, there is a constant $C_{n}$ such that if integer $k \geq \kappa_{n}:=2(n-1)+$ $\left(\eta_{1}+\eta_{2}\right) / \eta_{0}$, then

$$
P_{G / K}(\lambda, k, a) \leq C_{n} \min \left(1,\|\lambda\|^{\eta_{0}(2(n-1)-k)+\eta_{1}+\eta_{2}}\right) .
$$

(ii) Suppose $n=2, m=\min \left(\eta_{0}, \eta_{1}+\eta_{2}\right)$ and $M=\max \left(\eta_{0}, \eta_{1}+\eta_{2}\right)$. Then if integer $k \geq \kappa_{2}=1+M / 2 m$,

$$
P_{G / K}(\lambda, k, a) \leq C_{2} \min \left(1, \max \left(\|\lambda\|^{2 m(1-k)+M}\right) .\right.
$$

Proof. As noted previously, we obviously have $P_{G / K}(\lambda, k, a)$ uniformly bounded when $\|\lambda\| \leq 1$. Moreover, when $n \geq 3$ and integer $k \geq \kappa_{n}$, then $\eta_{0}(2(n-1)-k)+$ $\eta_{1}+\eta_{2} \leq 0$ and when $k \geq \kappa_{2}, 2 m(1-k)+M \leq 0$. Thus the task is to check that $P_{G / K}(\lambda, k, a) \leq C_{n}\|\lambda\|^{\eta_{0}(2(n-1)-k)+\eta_{1}+\eta_{2}}$ when $n \geq 3$ and the corresponding statement of (ii) when $n=2$.

Our proof of (i) will proceed by induction on $n$. We will leave the arguments for the base case until the end when it will be done in conjunction with the proof of (ii).

We will give the proof for type $B C_{n}$, but the modifications for the other types are essentially notational. For the induction argument, it will is natural to write $P_{n}(\lambda, k, a)$ rather than $P_{G / K}(\lambda, k, a)$ when the rank of $G / K$ is $n$.

Let $a \in A_{0}$. Since $\Phi^{+}(w(a))$ is a proper root subsystem, in bounding $P_{n}(\lambda, k, a)$ we may as well assume $\Phi^{+}(w(a))=\Psi^{+}$, where $\Psi$ is one of the finitely many maximal root subsystems, and that $w=i d$.

The maximal root subsystems of a symmetric space of Lie type $B C_{n}$ are: (a) Lie type $B C_{n-1}$, (b) Lie type $A_{n-1}$ and (c) Lie types $B C_{n-j} \times A_{j-1}$ with $n-j \geq 1$, $j \geq 2$.

Any spherical representation in $B C_{n}$ can be written as $\lambda=\sum_{i=1}^{n} \lambda_{i} e_{i}$ where $\lambda_{i}$ are non-increasing, non-negative integers. Thus $\lambda_{1} \leq\|\lambda\| \leq n \lambda_{1}$ and, consequently,

$$
\prod_{\alpha \in \Phi^{+}}|1+\langle\lambda, \alpha\rangle|^{m_{\alpha}} \leq C \lambda_{1}^{2\left(\begin{array}{c}
n \\
2
\end{array}\right) \eta_{0}+n\left(\eta_{1}+\eta_{2}\right)} .
$$

We now consider the three cases of maximal annihilating root subsystems separately.

Case (a) $\Psi$ is of type $B C_{n-1}$ : That means there is some index $n_{0} \in\{1, \ldots, n\}$ such that

$$
\Psi^{+}=\left\{e_{i} \pm e_{j}, e_{k}, 2 e_{k}: 1 \leq i<j \leq n, i, j, k \neq n_{0}\right\},
$$

and hence

$$
\left(\Psi^{+}\right)^{c}=\left\{e_{n_{0}} \pm e_{j}, e_{n_{0}}, 2 e_{n_{0}}: j \neq n_{0}\right\}
$$

(where $e_{n_{0}}-e_{j}$ should be replaced by $e_{j}-e_{n_{0}}$ if $j<n_{0}$ ). 
If $n_{0}=1$, then as $1+\left\langle\lambda, e_{1}+e_{j}\right\rangle \geq \lambda_{1}$ for all $j=2, \ldots, n$ and $1+\left\langle\lambda,(2) e_{1}\right\rangle \geq \lambda_{1}$, we see that

$$
\prod_{\alpha \in\left(\Psi^{+}\right)^{c}}|1+\langle\lambda, \alpha\rangle|^{m_{\alpha}} \geq \lambda_{1}^{(n-1) \eta_{0}+\eta_{1}+\eta_{2}}
$$

Thus for such $a$,

$$
P_{n}(\lambda, k, a) \leq \lambda_{1}^{(n-1) \eta_{0}(n-k)+\left(\eta_{1}+\eta_{2}\right)(n-k)}
$$

and that's dominated by the right hand side of (4.3) when $k \geq \kappa_{n}$.

So assume $n_{0} \neq 1$. Here we will use an induction argument assuming the statement holds for $n-1$. (Actually, all we will need to inductively assume is that $P_{n-1}(\lambda, k, a)$ is uniformly bounded for $k \geq \kappa_{n}$ and the claims of the lemma certainly ensure this.)

We consider the root subsystem

$$
\Phi^{\prime}=\left\{e_{i} \pm e_{j}, e_{k}, 2 e_{k}: 2 \leq i \neq j \leq n, 2 \leq k \leq n\right\} \subseteq \Phi,
$$

with the same multiplicities. This can be viewed as the restricted root system of the same Cartan class as $G / K$, but with rank $n-1$. For instance, if $G / K$ is of Cartan class $A I I I$, so that

$$
G / K=S U(p, n) / S U(p) \times S U(n)
$$

for some $p>n$, then $\Phi^{\prime}$ is the restricted root system of the symmetric space

$$
S U(p-1, n-1) / S U(p-1) \times S U(n-1),
$$

of Cartan class $A I I I$, Lie type $B C_{n-1}$. For the purposes of this proof, we will call this the 'reduced symmetric space'. We remark that the reduced symmetric space has rank $n-1$ and that the multiplicities of the roots are unchanged.

By identifying $a \in A_{0}$ with $X_{a} \in \mathfrak{a}$, we can assume $a=\sum_{i=1}^{n} a_{i} e_{i}$. We let $a^{\prime}=\sum_{i=2}^{n} a_{i} e_{i}$ (understood, appropriately, as an element in the reduced symmetric space) and observe that the annihilating root system of $a^{\prime}$ is of type $B C_{n-2}$.

Put $\lambda^{\prime}=\sum_{i=2}^{n} \lambda_{i} e_{i}$, so that for $\alpha \in \Phi^{\prime},\left\langle\alpha, \lambda^{\prime}\right\rangle=\langle\alpha, \lambda\rangle$. An elementary, but useful, observation is that $\Phi^{+}(a)^{c}$ consists of the union of the non-annihilating positive roots of $a$ that belong to $\Phi^{\prime}$ together with those non-annihilating positive roots that do not belong to $\Phi^{\prime}$, namely $e_{1} \pm e_{n_{0}}$. Moreover, the non-annihilating roots which are in $\Phi^{\prime}$ are precisely the non-annihilating roots of $a^{\prime}$. Thus

$$
P_{n-1}\left(\lambda^{\prime}, k, a^{\prime}\right)=\prod_{\alpha \in\left(\Psi^{+}\right)^{c} \cap \Phi^{\prime+}}|1+\langle\lambda, \alpha\rangle|^{-m_{\alpha} k} \prod_{\alpha \in \Phi^{\prime+}}|1+\langle\lambda, \alpha\rangle|^{m_{\alpha}} .
$$

Since $\left\langle\lambda, e_{1}+e_{n_{0}}\right\rangle \geq c \lambda_{1}$ and the induction assumption ensures that $P_{n-1}\left(\lambda^{\prime}, k, a^{\prime}\right)$ is bounded independently of $\lambda^{\prime}$ and $k$, we see that

$$
\begin{aligned}
P_{n}(\lambda, k, a) & =P_{n-1}\left(\lambda^{\prime}, k, a^{\prime}\right) \prod_{\alpha \in\left(\Psi^{+}\right)^{c} \backslash \Phi^{\prime+}}|1+\langle\lambda, \alpha\rangle|^{-m_{\alpha} k} \prod_{\alpha \in \Phi^{+} \backslash \Phi^{\prime+}}|1+\langle\lambda, \alpha\rangle|^{m_{\alpha}} \\
& \leq P_{n-1}\left(\lambda^{\prime}, k, a^{\prime}\right) \prod_{\alpha=e_{1} \pm e_{n_{0}}}|1+\langle\lambda, \alpha\rangle|^{-m_{\alpha} k} \prod_{\substack{\alpha=e_{1} \pm e_{j}, j=2, \ldots, n \\
e_{1}, 2 e_{1}}}|1+\langle\lambda, \alpha\rangle|^{m_{\alpha}} \\
(4.7) & \leq P_{n-1}\left(\lambda^{\prime}, k, a^{\prime}\right) \lambda_{1}^{\eta_{0}(2(n-1)-k)+\eta_{1}+\eta_{2}} \leq C \lambda_{1}^{\eta_{0}(2(n-1)-k)+\eta_{1}+\eta_{2}} .
\end{aligned}
$$

Case (b) $\Psi$ is of type $A_{n-1}$ : Hence $\Psi^{+}=\left\{s_{i} e_{i}-s_{j} e_{j}: 1 \leq i<j \leq n\right\}$ for some choice of $s_{i}= \pm 1$. We define $\Phi^{\prime}, a^{\prime}, \lambda^{\prime}$ as above, so that $\Phi^{\prime}$ is type $B C_{n-1}$ and the 
subset of annihilating roots of $a^{\prime}$ is of type $A_{n-2}$. Again we factor $P_{n}(\lambda, k, a)$ and use the fact that $P_{n-1}\left(\lambda^{\prime}, k, a^{\prime}\right)$ is bounded to see that

$$
\begin{aligned}
P_{n}(\lambda, k, a) & =P_{n-1}\left(\lambda^{\prime}, k, a^{\prime}\right) \prod_{\alpha \in\left(\Psi^{+}\right)^{c} \backslash \Phi^{\prime+}}|1+\langle\lambda, \alpha\rangle|^{-m_{\alpha} k} \prod_{\substack{\alpha \in \Phi^{+} \backslash \Phi^{\prime+}\\
}}|1+\langle\lambda, \alpha\rangle|^{m_{\alpha}} \\
& \leq P_{n-1}\left(\lambda^{\prime}, k, a^{\prime}\right) \prod_{\substack{\alpha=s_{1} e_{1}+s_{j} e_{j},(2) e_{1}}}|1+\langle\lambda, \alpha\rangle|^{-m_{\alpha} k} \prod_{\substack{\alpha=\varepsilon_{1} \pm e_{j},(2) e_{1}}}|1+\langle\lambda, \alpha\rangle|^{m_{\alpha}} \\
& \leq \prod_{\alpha=s_{1} e_{1}+s_{j} e_{j},(2) e_{1}}|1+\langle\lambda, \alpha\rangle|^{-m_{\alpha} k} \cdot \lambda_{1}^{2(n-1) \eta_{0}+\eta_{1}+\eta_{2}} .
\end{aligned}
$$

There is no loss in assuming $s_{1}=1$, thus

$$
\prod_{\alpha=s_{1} e_{1}+s_{j} e_{j},(2) e_{1}}|1+\langle\lambda, \alpha\rangle|^{-m_{\alpha} k} \leq \lambda_{1}^{-\#\left\{j>1: s_{j}=1\right\} \eta_{0} k} \lambda_{1}^{-\left(\eta_{1}+\eta_{2}\right) k} .
$$

If there is at least one $j>1$ such that $s_{j}=1$, then we have

$$
P_{n}(\lambda, k, a) \leq C \lambda_{1}^{\eta_{0}(2(n-1)-k)+\left(\eta_{1}+\eta_{2}\right)(1-k)},
$$

agreeing with (4.3).

So assume $s_{j}=-1$ for all $j>1$. We note that if $\alpha=e_{1}-e_{j}$, then $|1+\langle\lambda, \alpha\rangle|=$ $1+\lambda_{1}-\lambda_{j}$, so if there is some $j$ with $\lambda_{j} \leq \lambda_{1} / 2$, then $|1+\langle\lambda, \alpha\rangle| \geq \lambda_{1} / 2$. Hence

$$
\prod_{\alpha \in s_{1} e_{1}+s_{j} e_{j},(2) e_{1}}|1+\langle\lambda, \alpha\rangle|^{-m_{\alpha} k} \leq C \lambda_{1}^{-\eta_{0} k-\left(\eta_{1}+\eta_{2}\right) k}
$$

and we can obtain the same bound on $P_{n}(k, \lambda, a)$ as in (4.8) (with a different choice of constant).

Thus we can also assume $\lambda_{j} \geq \lambda_{1} / 2$ for all $j>1$. Then we give a direct argument, rather than appealing to induction. The choice of $s_{1}=1$ and $s_{j}=-1$ for all $j \neq 1$ means that

$$
\Phi^{+}(a)^{c}=\left\{e_{1}-e_{k}, e_{i}+e_{j},(2) e_{t}: 2 \leq i<j \leq n, k \geq 2, t \geq 1\right\} .
$$

Furthermore, $\left|1+\left\langle\lambda, e_{i}+e_{j}\right\rangle\right| \geq \lambda_{i}+\lambda_{j} \geq \lambda_{1}$ for all $i, j \geq 2$ and similarly $\left|1+\left\langle\lambda,(2) e_{t}\right\rangle\right| \geq$ $\lambda_{1} / 2$ for all $t \geq 1$. Thus

$$
\prod_{\alpha \in\left(\Psi^{+}\right)^{c}}|1+\langle\lambda, \alpha\rangle|^{m_{\alpha}} \geq \lambda_{1}^{\left(\begin{array}{c}
n-1 \\
2
\end{array}\right) \eta_{0}+n\left(\eta_{1}+\eta_{2}\right)} .
$$

Coupled with (4.5), this gives

$$
P_{n}(\lambda, k, a) \leq C \lambda_{1}^{\eta_{0}\left(2\left(\begin{array}{c}
n \\
2
\end{array}\right)-k\left(\begin{array}{c}
n-1 \\
2
\end{array}\right)\right)+n\left(\eta_{1}+\eta_{2}\right)(1-k)} .
$$

It is routine to check that this implies that the claim of the lemma holds.

Case (c) $\Psi$ is of type $B C_{n-j} \times A_{j-1}$ with $2 \leq j \leq n-1$ : In this situation, there are disjoint sets of indices, $I, J \subseteq\{1, \ldots, n\}$ where $|I|=n-j,|J|=j \geq 2$, and a choice $s_{t}= \pm 1$ for $t \in J$ such that

$$
\Psi^{+}=\left\{e_{i} \pm e_{j},(2) e_{t}: i<j, t \in I\right\} \cup\left\{s_{i} e_{i}-s_{j} e_{j}: i<j \in J\right\} .
$$

We set up the usual induction/factoring argument. If $1 \in I$, then set of annihilating roots of $a^{\prime}$ is type $B C_{n-j-1} \times A_{j-1}$ in the reduced symmetric space of type $B C_{n-1}$ (or type $A_{n-2}$ in $B C_{n-1}$ if $j=n-1$ ). Under this assumption, $\left(\Psi^{+}\right)^{c} \backslash \Phi^{\prime}$ 
contains all the roots $a=e_{1}+e_{j}$ for $j \in J$, and for such $\alpha$ we have $\langle\alpha, \lambda\rangle \geq \lambda_{1}$. As $|J| \geq 2$

$$
P_{n}(\lambda, k, a) \leq C \lambda_{1}^{-k \eta_{0}|J|+2(n-1) \eta_{0}+\eta_{1}+\eta_{2}} \leq C \lambda_{1}^{\eta_{0}(2(n-1)-2 k)+\eta_{1}+\eta_{2}},
$$

a better bound than (4.3).

Otherwise, $1 \in J$, so the set of annihilating roots of $a^{\prime}$ is type $B C_{n-j} \times A_{j-2}$ in type $B C_{n-1}$ (or $B C_{n-2}$ in $B C_{n-1}$ if $|J|=2$ ). Then $\left(\Psi^{+}\right)^{c} \backslash \Phi^{\prime}$ contains the roots $a=e_{1}+e_{i}$ for $i \in I$ and (2) $e_{1}$, hence the usual arguments gives

$$
\begin{aligned}
P_{n}(\lambda, k, a) & \leq C \lambda_{1}^{-k \eta_{0}|I|-k\left(\eta_{1}+\eta_{2}\right)+2(n-1) \eta_{0}+\eta_{1}+\eta_{2}} \\
& \leq C \lambda_{1}^{\eta_{0}(2(n-1)-k)+\left(\eta_{1}+\eta_{2}\right)(1-k)} .
\end{aligned}
$$

This completes the induction step.

We have seen that to start the induction argument we need only prove that $P_{2}(\lambda, k, a)$ is uniformly bounded for $k \geq \kappa_{3}$. Since $k \geq \kappa_{3}$ ensures that $2 m(1-k)+$ $M \leq 0$, we need only prove (4.4) to see this. For $B C_{2}$, we have $\Phi^{+}=\left\{e_{1} \pm e_{2}\right.$, (2) $\left.e_{1},(2) e_{2}\right\}$, thus

$$
\prod_{\alpha \in \Phi^{+}}|1+\langle\lambda, \alpha\rangle|^{m_{\alpha}} \leq C \lambda_{1}^{\eta_{0}}\left(1+\lambda_{1}-\lambda_{2}\right)^{\eta_{0}} \lambda_{1}^{\eta_{1}+\eta_{2}} \lambda_{2}^{\eta_{1}+\eta_{2}} .
$$

The maximal root subsystems of type $B C_{2}$ are of type $B C_{1}$ with positive roots either $(2) e_{1}$ or $(2) e_{2}$, or of type $A_{1}$ with the positive root being either $e_{1}-e_{2}$ or $e_{1}+e_{2}$. We can analyze each of these cases separately, using the fact that $\lambda_{1}-\lambda_{2} \sim \lambda_{1}$ if $\lambda_{2} \leq \lambda_{1} / 2$, and $\lambda_{2} \sim \lambda_{1}$ if $\lambda_{2} \geq \lambda_{1} / 2$. The details are left for the reader.

Proof. [of Theorem 3 First, suppose $G / K$ is Lie type $A_{n-1}$ or $D_{n}$. In the notation of Lemma 2 we have

$$
\begin{aligned}
\left\|\nu_{a}^{k}\right\|_{2}^{2} & \leq C \int_{\overline{\mathfrak{a}^{*+}}}\left(\left|\phi_{\lambda}(a)\right|^{k}|c(\lambda)|^{-1}\right)^{2} d \lambda \leq C \int \min \left(1,\|\lambda\|^{-\eta_{0} p_{k}}\right) d \lambda \\
& \leq C \int_{1}^{\infty} t^{-\eta_{0} p_{k}} t^{n-1} d t .
\end{aligned}
$$

Of course, the last integral is finite if $k$ is chosen so that $\eta_{0} p_{k}>n$. Using the values obtained for $p_{k}$ in the Lemma gives the specified choice of $k_{G}$.

A similar argument can be applied for types $B_{n}, C_{n}$ or $B C_{n}$, using the claims of Lemma 3

To prove the statement about the convolution of $k$ (possibly distinct) orbital measures $\nu_{a_{i}}$, with $a_{i} \in A_{0}$, we use the fact that $\nu_{a_{i}}^{k} \in L^{2}$ for the specified choices of $k$ and apply the generalized Holder's inequality in the same manner as we did in the proof of Corollary 2

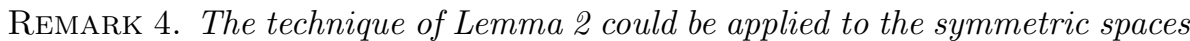
of type $B_{n}$ or $C_{n}$ which have the additional property that all restricted roots have the same multiplicity. But the results are no better than can be obtained by Lemma

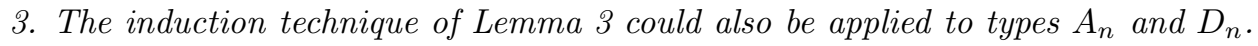
This takes much more work than Lemma 2 and gives only modest improvements.

Similar techniques can also be applied to the symmetric spaces with root systems of exceptional types. 
Proposition 2. Suppose $G / K$ is a symmetric space with restricted root system of exceptional Lie type $G_{2}, F_{4}, E_{6}, E_{7}$ or $E_{8}$. Then $\nu_{a_{1}} * \cdots * \nu_{a_{k}} \in L^{2}$ if $k \geq k_{G}$ as stated in the chart.

$\begin{array}{cccc}\text { Lie Type } & k_{G} & F_{4}-\text { Cartan Type } & k_{G} \\ E_{7}, E_{8} & 8 & E I I & 7 \\ G_{2} & 4 & E V I & 11 \\ E_{6}, F_{4} \text { all same mult } & 7 & E I X & 19\end{array}$

REMARK 5. For comparison, the dimension of $G / K$ is 40 for EII, 64 for EVI and 112 for EIX.

Proof. When all the restricted roots of the symmetric space have the same multiplicity, we reason as in the proof of Lemma 2, using the fact (with the notation of that lemma) shown in 17 that $s=1 /(n-1)$ if the Lie type is $E_{n}, s=1 / 5$ for type $F_{4}$ and $s=2 / 5$ for type $G_{2}$.

For the final three cases (Lie type $F_{4}$, Cartan types EII, EVI or EIX) we note that the maximal annihilating root systems are types $A_{1} \times A_{2}, A_{1} \times B_{2}, A_{1} \times C_{2}$, $A_{1} \times A_{1} \times A_{1}, B_{3}$ and $C_{3}$, all of which have cardinality at most 9 , and do a counting argument similar to that done in the proof of Theorem 2 ,

REMARK 6. It would be interesting to know the sharp $L^{2}$ results and whether the $L^{1}-L^{2}$ dichotomy only fails for the symmetric space of Lie type $A_{1}$ and Cartan class $A I$.

4.2. Differentiability properties. If $\nu^{k} \in L^{2}$, then $\nu^{2 k}=\nu^{k} * \nu^{k}$ has a continuous density function. However, more can be said about the smoothness of these measures using following fact proven in [4, Prop. 3.1(vi)]:

$$
\mid \frac{d^{m}}{d t^{m}}\left(\left.\phi_{\lambda}(g \exp (t X))\right|_{t=0} \mid \leq C_{1}(1+\|\lambda\|)^{m} .\right.
$$

In proving Theorems 2 and 3, we have seen that there are constants $C$ and $q(k)$ such that $\left|\left(\phi_{\lambda}(a)\right)^{k} c(\lambda)^{-1}\right|^{2} \leq C \min \left(1,\|\lambda\|^{q(k)}\right)$ for all $\lambda$. Thus, with $n=\operatorname{rank} G / K$, we have

$$
\begin{aligned}
\int_{\mathfrak{a}^{*}} \mid \phi_{\lambda}(a)^{k} \frac{d}{d t}\left(\left.\phi_{\lambda}(g \exp (t X))\right|_{t=0}|c(\lambda)|^{-2} \mid d \lambda\right. & \leq C \int_{\overline{\mathfrak{a}^{*+}}}\left|\phi_{\lambda}(a)^{k / 2} c(\lambda)^{-1}\right|^{2}(1+\|\lambda\|) \\
& \leq C \int_{\overline{\mathfrak{a}^{*+}}}\|\lambda\|^{q(k / 2)}(1+\|\lambda\|) \\
& \leq C \int_{1}^{\infty} t^{n-1+q(k / 2)+1}
\end{aligned}
$$

and this is finite provided $n+q(k / 2)<-1$. If $k$ is chosen sufficiently large that this inequality holds, then Leibniz's rule applied to the Inversion formula (19. IV Thm. 7.5]) shows that

$$
X \nu_{a}^{k}(g)=\frac{1}{|W|} \int_{\mathfrak{a}^{*}} \phi_{\lambda}(a)^{k} \frac{d}{d t}\left(\left.\phi_{\lambda}(g \exp (t X))\right|_{t=0}|c(\lambda)|^{-2} d \lambda\right.
$$

is well defined and hence $\nu_{a}^{k}$ is differentiable. More generally, $\nu_{a}^{k}$ is $r$-times differentiable if $n-1+q(k / 2)+r<-1$.

For example, if $G / K$ is Lie type $A_{n}$, then Lemma2 2 yields $q(k / 2) \leq \eta_{0}(n-k / 2)$. Thus we have $\nu_{a}^{k}$ is differentiable for all $a \in A_{0}$ if $k>2 n+2(n+1) / \eta_{0}$. If $a$ is a 
regular element and $G / K$ is not of Lie type $A_{1}$ and Cartan type $A I$, then one can similarly use Lemma1 to check that $\nu_{a}^{k}$ is differentiable if $k>4$. Similar statements can be made about higher orders of differentiability. These observations improve

upon $[2]$ where it was shown that if $a$ was a regular element, then $\nu_{a}^{k}$ is differentiable for $k>\operatorname{dim} G / K+1$.

\section{References}

[1] M. Abramowitz and I. Stegun, Handbook of mathematical functions, National bureau of standards, 1970.

[2] B. Anchouche, Regularity of the Radon-Nikodym derivative of a convolution of orbital measures on noncompact symmetric spaces, preprint 2018.

[3] B. Anchouche, S.K. Gupta and A. Plagne, Orbital measures on $S U(2) / S O(2)$, Monatsh. Math., 178(2015), 493-520.

[4] J. P. Anker, The spherical Fourier transform of rapidly decreasing functions. A simple proof of a characterization due to Harish-Chandra, Helgason, Trombi and Varadarajan, J. Func. Anal., 96(1991), 331-349.

[5] M. Caselle and U. Magnea, Random matrix theory and symmetric spaces, Physics reports 394(2004), 41-156.

[6] J.J. Duistermaat, J.A.C. Kolk and V.S. Varadarajan, Functions, flows and oscillatory integrals on flag manifolds and conjugacy classes in semisimple Lie groups, Comp. Math. 49(1983), 309-398.

[7] A. Fitouhi and M. Hamza, A uniform expansion for the eigenfunction of a singular second order differential operator, Siam J. Math. Anal., 21(1990), 1619-1632.

[8] P. Graczyk and P. Sawyer, Absolute continuity of convolutions of orbital measures on Riemannian symmetric spaces, J. Func. Anal., 259(2010), 1759-1770.

[9] P. Graczyk and P. Sawyer, A sharp criterion for the existence of the product formula on symmetric spaces of type $A_{n}$, J. Lie Theory 20(2010), 751-766.

[10] P. Graczyk and P. Sawyer, Convolution of orbital measures on symmetric spaces of type $C_{p}$ and $D_{p}$, J. Aust. Math. Soc. 98(2015), 232-256.

[11] P. Graczyk and P. Sawyer, Convolution of orbital measures on symmetric spaces: a survey, Contemporary Math 668(2016), 81-110.

[12] S. Gupta and K.E. Hare, $L^{2}$-singular dichotomy for orbital measures of classical compact Lie groups, Adv. Math., 222(2009), 1521-1573.

[13] S.K. Gupta, K.E. Hare and S. Seyfaddini, $L^{2}$-singular dichotomy for orbital measures of classical simple Lie algebras, Math Zeit. 262(2009), 91-124.

[14] S.K. Gupta and K.E. Hare, The absolute continuity of convolutions of orbital measures in symmetric spaces, J. Math. Anal. and Appl., 450(2017), 81-111.

[15] K.E. Hare and P. Skoufranis, The smoothness of orbital measures on exceptional Lie groups and algebras, J. Lie Theory, 21(2011), 987-1007.

[16] K.E. Hare, D. Wilson and W.L. Yee, Pointwise estimates of the size of characters of compact Lie groups, J. Aust. Math. Soc., 69(2000), 61-84.

[17] K.E. Hare and K. Yeats, The size of characters of exceptional Lie groups, J. Aust. Math. Soc., $77(2004), 233-248$.

[18] S.Helgason, Differential geometry, Lie groups and symmetric spaces, Academic Press, New York, 1978.

[19] S. Helgason, Groups and Geometric analysis, Academic Press, Florida, 1984.

[20] J. Humphreys, Introduction to Lie algebras and representation theory, Springer Verlag, New York, 1994.

[21] D. Ragozin, Zonal measure algebras on isotropy irreducible homogeneous spaces, J. Func. Anal., 17(1974), 355-376.

[22] J. Wolf, Harmonic analysis on commutative spaces, AMS Mathematical surveys and monographs 142, 2007. 


\section{Appendix}

In the charts below we summarize some of the important facts about these symmetric spaces. These are taken from [5] and [18, Ch. X].

\begin{tabular}{|ccccc|}
\hline Restricted & Cartan & $G / K$ & $\operatorname{dim} G / K$ & Multiplicities \\
root space & class & $\frac{S L(n, R)}{S O(n)}$ & $\frac{1}{2}(n-1)(n+2)$ & $\eta_{0} ; \eta_{1} ; \eta_{2}$ \\
$A_{n-1}$ & $A I$ & $\frac{S L(n, H)}{S p(n)}$ & $(n-1)(2 n+1)$ & $4 ; 0 ; 0$ \\
$A_{n-1}$ & $A I I$ & $\frac{S U(p, n)}{S U(p) \times S U(n)}$ & $2 p n$ & $2 ; 1 ; 2(p-n)$ \\
$B C_{n}, p>n$ & $A I I I$ & $\frac{S p(n, R)}{S U(n)}$ & $n(n+1)$ & $1 ; 1 ; 0$ \\
$C_{n}, p=n$ & $C I$ & $\frac{S p(p, n)}{S p(p) \times S p(n)}$ & $4 p n$ & $4 ; 3 ; 4(p-n)$ \\
$C_{n}$ & $C I I$ & $\frac{S O^{*}(4 n)}{U(2 n)}$ & $2 n(2 n-1)$ & $4 ; 1 ; 0$ \\
$B C_{n}, p>n$ & $\frac{S O^{*}(4 n+2)}{U(2 n+1)}$ & $2 n(2 n+1)$ & $4 ; 1 ; 4$ \\
$C_{n}, p=n$ & $D I I$ (even) & & $1 ; 0 ; p-n$ \\
$C_{n}$ & $D I I$ (odd) & $\frac{S O_{0}(p, n)}{S O(p) \times S O(n)}$ & $p n$ & \\
$B C_{n}$ & $D I I$ & & \\
$B_{n}, p>n$ & $B D I$ & $D_{n}, p=n$ & &
\end{tabular}

\begin{tabular}{cccc|} 
Restricted & Cartan class & $\operatorname{dim} G / K$ & Multiplicities \\
root space & $E I I I$ & 32 & $8 ; 6 ; 1$ \\
$B C_{2}$ & $E I V$ & 26 & 8 \\
$A_{2}$ & $E V I I$ & 54 & $8 ; 1$ \\
$C_{3}$ & $F I I$ & 16 & $8 ; 7$ \\
$B C_{1}$ & & \\
\hline
\end{tabular}

\begin{tabular}{cccc|} 
Restricted & Cartan class & $\operatorname{dim} G / K$ & Multiplicities \\
$G_{2}$ & $G$ & 8 & 1 \\
& $E I I$ & 40 & 1,2 \\
$F_{4}$ & $E V I$ & 64 & 1,4 \\
& $E I X$ & 112 & 8,1 \\
$E_{6}$ & $F I$ & 28 & 1 \\
$E_{7}$ & $E I$ & 42 & 1 \\
$E_{8}$ & $E V I I I$ & 70 & 1 \\
\end{tabular}

Dept. of Mathematics, Sultan Qaboos University, P.O.Box 36 Al Khodh 123, SulTANATE OF OMAN

E-mail address: gupta@squ.edu.om

Dept. of Pure Mathematics, University of Waterloo, Waterloo, Ont., Canada, N2L 3G1

E-mail address: kehare@uwaterloo.ca 